\title{
Mipomersen: evidence-based review of its potential in the treatment of homozygous and severe heterozygous familial hypercholesterolemia
}

\author{
This article was published in the following Dove Press journal: \\ Core Evidence \\ 30 May 2012 \\ Number of times this article has been viewed
}

\author{
Klaus G Parhofer \\ Medical Department II, Grosshadern, \\ University Munich, Munich, Germany
}

\begin{abstract}
Familial hypercholesterolemia (FH) is an autosomal-dominant inherited disease with a prevalence of one in 500 (heterozygous) to one in 1,000,000 (homozygous). Mutations of the low-density lipoprotein (LDL) receptor gene, the apolipoprotein B100 gene, or the PCSK9 gene may be responsible for the disease. The resulting LDL hypercholesterolemia results in premature atherosclerosis as early as childhood (homozygous FH) or in adulthood (heterozygous FH). Current treatment modalities include lifestyle modification, combination drug therapy (statin-based), and apheresis. Mipomersen is an antisense oligonucleotide which inhibits apolipoprotein B production independent of LDL receptor function and thus works in homozygous FH, heterozygous FH, and other forms of hypercholesterolemia. Mipomersen is given $200 \mathrm{mg} /$ week subcutaneously. Phase III studies indicate that the LDL cholesterol concentration can be reduced by $25 \%-47 \%$, lipoprotein(a) levels by $20 \%-40 \%$, and triglyceride concentrations by approximately $10 \%$. In general, mipomersen has no effect on high-density lipoprotein cholesterol concentrations. Although there is considerable interindividual variability, the observed lipid effects are largely independent of age, gender, concomitant statin therapy, and underlying dyslipoproteinemia. The most common side effects are injection site reactions (70\%-100\%), flu-like symptoms (29\%-46\%), and elevated transaminases associated with an increased liver fat content $(6 \%-15 \%)$. Mipomersen may be an interesting addon drug in patients with heterozygous or homozygous FH not reaching treatment goals, either because baseline values are very high or because high-dose statins are not tolerated.
\end{abstract}

Keywords: antisense oligonucleotide, statin intolerance, apolipoprotein B

Core evidence clinical impact summary for mipomersen for familial hypercholesterolemia (FH)

\begin{tabular}{|c|c|c|}
\hline Outcome measure & Evidence & Implications \\
\hline \multicolumn{3}{|l|}{ Disease-oriented evidence } \\
\hline LDL-cholesterol reduction & Substantial & $\begin{array}{l}\text { In patients with homozygous or } \\
\text { heterozygous FH LDL-cholesterol reduction } \\
\text { of } 25 \%-47 \% \text { on top of maximally tolerated } \\
\text { drug therapy }\end{array}$ \\
\hline Lipoprotein(a) reduction & Substantial & $\begin{array}{l}\text { Approximately } 30 \% \text { lipoprotein(a) reduction; } \\
\text { Significant reduction of lipoprotein(a), not } \\
\text { achievable with currently available drugs }\end{array}$ \\
\hline $\begin{array}{l}\text { Patient-oriented evidence } \\
\text { Injection site reactions }\end{array}$ & Substantial & $\begin{array}{l}\text { Most patients (70-100\%) describe injection } \\
\text { site reaction }\end{array}$ \\
\hline
\end{tabular}

(Continued)
Correspondence: Klaus G Parhofer Medical Department II, Grosshadern, University Munich, Marchioninistraße 15, 8I377 Munich, Germany

Tel +498970953010

Fax +49897095 8879

Email klaus.parhofer@med.unimuenchen.de 


\begin{tabular}{|lll|}
\hline $\begin{array}{l}\text { (Continued) } \\
\text { Outcome measure }\end{array}$ & Evidence & Implications \\
\hline Flu-like symptoms & Moderate & $\begin{array}{l}\text { Approximately } 30 \% \text { of patients describe } \\
\text { flu-like symptoms (not significantly different } \\
\text { from placebo) } \\
\text { Elevated LFTs }\end{array}$ \\
Economic evidence & Moderate & $\begin{array}{l}6 \%-15 \% \text { of patients have elevated LFTs } \\
\text { The drug has not yet been approved by US } \\
\text { Abbreviations: LDL, low-density lipoprotein; LFT, liver function test; FDA, Food and Drug Administration. }\end{array}$ \\
\hline
\end{tabular}

\section{Familial hypercholesterolemia}

Familial hypercholesterolemia (FH) is an inherited disease, characterized by an autosomal-dominant mode of inheritance. Heterozygous $\mathrm{FH}$ has a prevalence of approximately one in 500 while the prevalence of homozygous $\mathrm{FH}$ is approximately one in 1,000,000 in Western Europe. The frequency may be considerably higher in selected populations with high consanguinity.

Mutations of the low-density lipoprotein (LDL) receptor gene are the underlying defect in the majority of $\mathrm{FH}$ patients, but mutations in two other genes can also cause $\mathrm{FH}$, ie, mutations in the apolipoprotein B100 gene and mutations in the pro-protein convertase subtilisin/kexin type 9 gene. The LDL receptor gene is located on chromosome 19, and more than 1000 mutations affecting the function of the receptor are described. ${ }^{1}$ The resulting defects refer to different functions, ie, ligand binding, transport, internalization, recycling, or total lack or receptors. ${ }^{1}$ Several types of LDL receptor mutation have been described, including premature stop codons, single amino acid substitutions, large rearrangements, mutations affecting the promoter region, and mutations affecting splicing of premessenger RNA. Clinically, the most severe forms of $\mathrm{FH}$ are related to total lack of receptors, while receptor-defective mutations usually lead to lesser symptoms.

Although it was initially thought that FH is characterized by an overproduction of lipoproteins, kinetic studies, and particularly the work of Brown and Goldstein, demonstrated that the underlying metabolic defect in FH is the absence of a high affinity receptor for uptake of LDL. ${ }^{2,3}$

Clinical FH can also be the result of ligand-defective apolipoprotein $\mathrm{B}$. In contrast with the many mutations described for the LDL receptor, there are only two mutations described for defective apolipoprotein B (both affecting ARG 3500). ${ }^{4,5}$ Finally, gain of function mutations of PCSK9 may also lead to clinical FH, because PCSK9 reduces hepatic LDL receptor activity. ${ }^{6}$ Interestingly, loss of function mutations are associated with lower LDL cholesterol concentrations and less atherosclerosis. ${ }^{7}$
FH is characterized by elevated plasma LDL cholesterol and apolipoprotein B levels, resulting in a severely increased risk for atherosclerosis. In untreated patients, clinical symptoms of atherosclerosis typically develop in the fourth or fifth decade of life. Other risk factors, such as smoking, hypertension, diabetes, and elevated lipoprotein(a) may change the clinical course.

\section{Current treatment options}

Current guidelines recommend lowering the LDL cholesterol concentration by at least $50 \%$ of baseline. ${ }^{8}$ Ideally, a LDL cholesterol level $<100 \mathrm{mg} / \mathrm{dL}$ should be achieved. Lifestyle modification, statins, ezetimibe, bile acid sequestrants, nicotinic acid, and fibrates are used to achieve this goal. Lifestyle modification aims at lowering LDL cholesterol and reducing other risk factors for cardiovascular disease. ${ }^{9}$ However, in patients with $\mathrm{FH}$, only a modest reduction of LDL cholesterol can be induced, even if the diet contains less than 7\% saturated fat and less than $200 \mathrm{mg}$ cholesterol per day. Although helpful and generally recommended, these means are rarely sufficient to achieve treatment goals. Therefore, drug therapy is required in almost all patients.

Statins can safely lower LDL cholesterol levels by up to $50 \%$, and there is overwhelming evidence that statins reduce cardiovascular morbidity and mortality as well as overall mortality. ${ }^{10,11}$ It has also been shown that the prognosis of patients with heterozygous and homozygous $\mathrm{FH}$ has been dramatically improved since the introduction of statins. ${ }^{12,13}$ While patients with heterozygous FH usually respond very well to statin treatment, the response rate in homozygous FH is usually less predictable. Depending on the underlying defect, LDL cholesterol reductions between $0 \%$ and $50 \%$ are observed in such patients. Observational data from large cohorts suggest that long-term statin treatment reduces the risk of cardiovascular disease considerably. ${ }^{14}$

However, treatment goals cannot be achieved by statin therapy in many patients, or statin therapy is contraindicated or poorly tolerated. Ezetimibe, bile acid sequestrants, nicotinic acid, and fibrates may be used in this situation, either 
as addon therapy to statins or as monotherapy. The most potent combination is probably high-dose statins combined with ezetimibe and bile acid sequestrants. ${ }^{15}$ However, even if combination therapy is performed at maximal doses, LDL cholesterol reduction rarely exceeds $60 \%$.

Another option to decrease LDL cholesterol is regular apheresis or plasmapheresis. This treatment modality has been in use for more than 30 years. ${ }^{16,17} \mathrm{~A}$ single apheresis usually decreases LDL cholesterol by approximately $70 \%$, resulting in a mean LDL cholesterol reduction of approximately $30 \%$.

However, if regular apheresis is performed at weekly intervals, LDL cholesterol does not rebound to the original baseline value, resulting in an average LDL cholesterol concentration of approximately $50 \%$ of the original baseline value. Several studies indicate that $\mathrm{FH}$ patients treated with regular apheresis benefit from such treatment. ${ }^{16}$ Recommendations concerning when to initiate such treatment differ considerably between countries. In Japan and Germany, where regular apheresis is relatively widely used, patients with established coronary heart disease and LDL cholesterol above goal may qualify for such treatment. In the US, apheresis is only recommended in patients with $\mathrm{FH}$ and LDL cholesterol $>300 \mathrm{mg} / \mathrm{dL}$ (without coronary heart disease) or $>200 \mathrm{mg} / \mathrm{dL}$ (with coronary heart disease).${ }^{18}$ Although regular apheresis is a time-consuming and cost-intensive form of therapy, it is generally well tolerated with very few side effects. Finally, liver transplantation can be used in selected cases of homozygous FH. This treatment option has been primarily used in pediatric patients sometimes as a combined heartliver transplant. ${ }^{19}$

\section{New therapeutic strategies}

A number of new therapeutic strategies (Table 1) to lower LDL cholesterol have been developed over recent years. ${ }^{20}$ These include inhibition of apolipoprotein B production, inhibition of PCSK9 production, monoclonal antibodies binding to PCSK9, thyroid mimetics, cholesterylester transfer protein (CETP) inhibitors, and microsomal triglyceride transfer protein (MTP) inhibitors. All of these approaches have been shown to reduce LDL cholesterol to varying degrees. Although this review primarily focuses on mipomersen, an antisense oligonucleotide for inhibition of production of apoprotein B, the other new approaches will also be briefly discussed.

\section{Strategies to lower PCSK9}

PCSK9 is an important regulator of LDL receptor degradation, because binding of PCSK9 to the LDL receptor results 
in degradation of the receptor, thereby preventing recycling of the receptor. Loss of function mutations of PCSK9 are associated with increased LDL receptor function, low LDL cholesterol, and reduced cardiovascular risk. ${ }^{7}$ PCSK 9 activity can be reduced by antisense oligonucleotides inhibiting PCSK9 production, by small interfering RNAs also inhibiting production, or by monoclonal antibodies. ${ }^{21-23}$ Interestingly, statins and fibrates increase PCSK9 expression, probably as part of a feedback mechanism following increased LDL receptor-mediated uptake of cholesterol during therapy with these drugs; therefore, combination therapy may be of particular potential to reduce LDL cholesterol levels. ${ }^{24}$ However, it should be noted that this approach will only work if functional LDL receptors are present, thus only in heterozygous $\mathrm{FH}$ and not in the homozygous form of the disease.

\section{Thyroid mimetics}

It is well known that hyperthyroidism is associated with low LDL cholesterol concentration, and hypothyroidism with hypercholesterolemia. A number of selective agonists to the thyroid receptor expressed in the liver have been developed. One of them, eprotirome, has been shown to decrease LDL cholesterol by approximately $30 \%$ if added to a statin. ${ }^{25}$ However, the development of eprotirome was recently stopped because of side effects in cartilage seen in animal studies. Whether this holds true for the other thyroid mimetics remains unclear.

\section{CETP inhibitors}

CETP is a key enzyme of lipid metabolism, and facilitates the transfer of cholesterol esters from high-density lipoprotein (HDL) particles to other lipoproteins. ${ }^{26}$ The inhibition of CETP results in an increase of HDL cholesterol and LDL cholesterol is decreased if torcetrapib, anacetrapib or evacetrapib are used, while dalcetrapib has no effect on LDL cholesterol levels. The development of torcetrapib has been stopped because of off-target effects (blood pressure increase) resulting in an unexpected increased mortality and morbidity, while the development of Dalcetrapib was stopped because of lack of efficacy. Anacetrapib is evaluated in a large outcome trial. If these outcome trials show benefit, then CETP inhibition may also be useful in patients with FH.

\section{MTP inhibition}

MTP is an enzyme necessary for the assembly of very-low-density lipoprotein. Mutations of MTP result in hypobetalipoproteinemia, so MTP inhibition was developed as a strategy to lower LDL cholesterol concentrations. ${ }^{27}$ MTP inhibition by lomitapide results in an LDL cholesterol reduction of approximately $30 \%$. MTP inhibition also induces an increase in hepatic steatosis, the importance of which is unclear. However, it should be noted that patients with hypobetalipoproteinemia also have hepatic steatosis and are still characterized by longevity.

\section{Mipomersen: antisense- oligonucleotide inhibiting apolipoprotein B}

Apolipoprotein B is the central protein of very-low-density lipoprotein, intermediate density lipoprotein, and LDL. Although epidemiological and interventional studies have focused on LDL-cholesterol, newer data indicate that apolipoprotein $\mathrm{B}$ concentrations may be a better predictor and target for atherosclerosis prevention. ${ }^{28}$ This also relates to the fact that apolipoprotein B containing lipoproteins only contain one molecule of apolipoprotein B, while the amount of cholesterol and cholesterol ester is to a certain point variable (leading to LDL size heterogeneity). Apolipoprotein B is essential for the production and catabolism of very-low-density lipoprotein and LDL. Without apolipoprotein B, these lipoproteins cannot be produced. Furthermore, apolipoprotein B100 is the main ligand for the LDL receptor.

Based on these observations, apolipoprotein B is an interesting target for atherosclerosis prevention. While most currently available approaches (statins, ezetimibe, bile acid sequestrants) primarily induce increased catabolism of apolipoprotein B-containing lipoproteins, another promising strategy would be to reduce the production of these lipoproteins. While MTP inhibition also interferes with the assembly and secretion of apolipoprotein B-containing lipoproteins, a much more direct approach is to reduce the production of apolipoprotein B100.

Mipomersen (previously ISIS-301012) is a 20-nucleotide second-generation antisense oligonucleotide targeting human apolipoprotein B100 that is complementary to a sequence within the coding region of human apolipoprotein B mRNA (exon 22, position 3249-3269 base pairs). ${ }^{29}$ Following the binding of the oligonucleotide to the mRNA, degradation by endogenous RNase $\mathrm{H}$ is induced. As a consequence, the synthesis of apolipoprotein B is reduced, resulting in decreased production and secretion of apolipoprotein B-containing lipoproteins. Production and secretion of apolipoprotein B48 from enterocytes, which is required to form chylomicrons, is not inhibited.

\section{Phase I studies}

The initial Phase I study was conducted as a double-blind, randomized, placebo-controlled, dose-escalation study in 
36 volunteers with mild dyslipidemia. ${ }^{30}$ Subjects were treated with weekly doses of mipomersen 50-400 mg subcutaneously. Apolipoprotein B and LDL cholesterol were reduced by up to $50 \%$ and $35 \%$, respectively. No significant changes in HDL cholesterol were observed. Apolipoprotein B and LDL cholesterol remained below baseline for up to 3 months after the last dose. The most common adverse event was erythema at the injection site in more than $70 \%$ of subjects.

In a second Phase I study, the interaction of mipomersen with simvastatin and ezetimibe was evaluated. ${ }^{31}$ This study confirmed that there are no clinically relevant interactions between these drugs in 10 healthy volunteers. In addition, it was shown that mipomersen does not inhibit the major cytochrome $\mathrm{P} 450$ isoform enzymes, which is consistent with the fact that antisense drugs are not metabolized through the cytochrome system.

\section{Phase II studies}

In a number of Phase II trials, mipomersen has been shown to result in prolonged, dose-dependent reduction of LDL cholesterol, apolipoprotein B, triglycerides, and lipoprotein(a), as shown in Table 2. In one study, 44 patients with heterozygous $\mathrm{FH}$ were enrolled and received doses ranging from $50 \mathrm{mg}$ to $300 \mathrm{mg}$ of mipomersen for up to 13 weeks. $^{32}$ LDL cholesterol was reduced by $21 \%$ in the $200 \mathrm{mg} /$ week group and $34 \%$ in the $300 \mathrm{mg} /$ week group, with a reduction in apolipoprotein $\mathrm{B}$ of $23 \%$ and $33 \%$, respectively. Given that all patients were on conventional lipid-lowering therapy (statins), this study shows that mipomersen has an incremental LDL cholesterol-lowering effect when added to conventional lipid-lowering therapy in FH patients.

Similarly, it was shown in a group of 74 patients with hypercholesterolemia receiving stable statin therapy that mipomersen induced significant changes from baseline in apolipoprotein B and LDL cholesterol at doses between
$100 \mathrm{mg}$ and $400 \mathrm{mg}$ per week over 13 weeks, while $30 \mathrm{mg}$ per week had no significant effect. ${ }^{33}$ After 13 weeks of dosing at $200 \mathrm{mg} /$ week, mipomersen reduced apolipoprotein B and LDL cholesterol by $36 \%$ and triglycerides by $15 \%$. HDL cholesterol did not change. Injection site reactions (erythema in $90 \%$ ) and hepatic transaminase increases (17\%) were the most common side effects, leading to discontinuation in three subjects.

The same authors also reported the effect of mipomersen in 50 subjects with mild to moderate hyperlipidemia, and showed that doses ranging from $50 \mathrm{mg} /$ week to $400 \mathrm{mg} /$ week resulted in a dose-dependent reduction in all apolipoprotein B-containing lipoproteins over a period of 13 weeks. ${ }^{34}$ In the $200 \mathrm{mg} /$ week and $300 \mathrm{mg} /$ week groups, mean reductions from baseline in LDL cholesterol were $45 \%$ and $61 \%$, corresponding to a decrease in apolipoprotein B concentration of $46 \%$ and $61 \%$, respectively. All subjects treated with mipomersen experienced injection site reactions and $18 \%$ showed transaminase elevations more than three times the upper limit of normal. To evaluate further the increase in liver function tests, intrahepatic triglyceride content was studied by model reference technology in a randomized, double-blind, placebo-controlled study in 21 patients with $\mathrm{FH}$ receiving weekly mipomersen doses of $200 \mathrm{mg}$ or placebo for 13 weeks while continuing statin therapy. Mipomersen reduced LDL cholesterol and apolipoprotein B by approximately $20 \%$. For the whole group, there was a trend towards an increase in intrahepatic triglyceride content, and one patient developed mild hepatic steatosis, which was reversible following discontinuation of mipomersen.

\section{Phase III studies}

Five randomized, double-blind, placebo-controlled Phase III studies using $200 \mathrm{mg}$ of mipomersen have been

Table 2 Dose-dependent effect of mipomersen on LDL-C, apolipoprotein B, and lipoprotein(a) in Phase II studies of 5-I3 weeks' duration (\% change compared with baseline)

\begin{tabular}{|c|c|c|c|c|c|c|c|c|c|}
\hline Subjects & Statins & $\mathbf{n}$ & Duration & $30 \mathrm{mg} / \mathrm{w}$ & $50 \mathrm{mg} / \mathrm{w}$ & $100 \mathrm{mg} / \mathrm{w}$ & $200 \mathrm{mg} / \mathrm{w}$ & $300 \mathrm{mg} / \mathrm{w}$ & $400 \mathrm{mg} / \mathrm{w}$ \\
\hline \multirow[t]{4}{*}{ Heterozygous FH } & Yes & 44 & $6 w$ & & & & & & \\
\hline & & & LDL-C & & $-13 \pm 15$ & $-11 \pm 10$ & $-21 \pm 23$ & $-34 \pm 18$ & \\
\hline & & & ApoB & & $-10 \pm 12$ & $-8 \pm 11$ & $-23 \pm 19$ & $-33 \pm 22$ & \\
\hline & & & $\operatorname{Lp}(a)$ & & $-3 \pm 10$ & $-15 \pm 10$ & $-17 \pm 19$ & $-24 \pm 26$ & \\
\hline \multirow[t]{3}{*}{ Hypercholesterolemia } & Yes & 74 & $5-13 w$ & & & & & & \\
\hline & & & LDL-C & $2 \pm 12$ & & $-21 \pm 10$ & $-27 \pm 21$ & $-52 \pm 14$ & $-38 \pm 31$ \\
\hline & & & ApoB & $0 \pm 12$ & & $-19 \pm 11$ & $-24 \pm 20$ & $-54 \pm 19$ & $-44 \pm 22$ \\
\hline Mild to moderate & No & 50 & $13 w$ & & & & & & \\
\hline \multirow[t]{2}{*}{ hyperlipidemia } & & & LDL-C & & -8 & -16 & -44 & -61 & -70 \\
\hline & & & ApoB & & -17 & -22 & -45 & -61 & $-7 \mid$ \\
\hline
\end{tabular}

Abbreviations: ApoB, apolipoprotein B; FH, familial hypercholesterolemia; LDL-C, low-density lipoprotein cholesterol; Lp(a), lipoprotein(a); w, week. 
completed..$^{35-39}$ Only one study is been published in full so far, and the others are only available in abstract form. In all Phase III studies, mipomersen $200 \mathrm{mg} /$ week was evaluated in patients with homozygous $\mathrm{FH}$ or heterozygous $\mathrm{FH}$ and with severe hypercholesterolemia, and in patients with statin intolerance. Except for the latter group, mipomersen was always coadministered with other lipid-lowering agents, including statins. The duration of all the Phase III studies was 26 weeks, and the primary and secondary efficacy endpoints were met in all cases (Table 3 ).

Mipomersen $200 \mathrm{mg} /$ week was tested in a multicenter study of 51 patients with homozygous FH on a low-fat diet and maximum tolerated lipid-lowering drugs. Thirty-four patients were assigned to mipomersen and 17 to placebo. LDL cholesterol (mean at baseline $11.4 \pm 3.6 \mathrm{mmol} / \mathrm{L}$ ) decreased by $24.7 \%$. Similarly, apolipoprotein B decreased by $26.8 \%$ and lipoprotein(a) by $31.1 \%$. In line with the Phase II studies, the most common adverse events were injection site reactions $(76 \%$ in the mipomersen group and $24 \%$ in the placebo group) and increases in liver function tests (12\% in the mipomersen group). ${ }^{36}$

In 124 adult patients with heterozygous $\mathrm{FH}$ and cardiovascular disease, 26 weeks of mipomersen $200 \mathrm{mg} /$ week resulted in an LDL cholesterol reduction of $34 \%$, an apolipoprotein B reduction of $26.3 \%$, and a lipoprotein(a) reduction of $20 \%$. Again, injection site reactions and flulike symptoms were the most common adverse events. Liver function tests increased to more than three times the upper limit of normal in $6 \%$ of patients treated with mipomersen. ${ }^{37}$

Another study evaluated mipomersen in 58 patients with severe FH. Mipomersen $200 \mathrm{mg} /$ week for 26 weeks reduced LDL cholesterol by $36 \%$ from a mean baseline level of $276 \mathrm{mg} / \mathrm{dL}$ and also significantly decreased apolipoprotein B and lipoprotein(a), with no change in HDL cholesterol. Side effects were similar as in other mipomersen studies, with injection site reactions (90\% in mipomersen group, $32 \%$ in placebo group) and flu-like symptoms (46\% in mipomersen group, $21 \%$ in placebo group) being the most common adverse events. In $15 \%$ of mipomersen-treated patients (but in none of the placebo patients) alanine aminotransferase was elevated to above three times the upper limit of normal. ${ }^{38}$

In a larger study in 158 adults with hypercholesterolemia and high cardiovascular risk, mipomersen $200 \mathrm{mg}$ / week for 26 weeks reduced LDL cholesterol by $37 \%$, with similar changes in apolipoprotein B and lipoprotein(a). In $50 \%$ of mipomersen-treated patients, LDL cholesterol was decreased to $<70 \mathrm{mg} / \mathrm{dL}$. Injection site reactions and flu-like symptoms were again the most common side effect. Fourteen percent of mipomersen-treated patients had alanine aminotransferase elevations above three times the upper limit of normal. ${ }^{35}$

Finally, mipomersen $200 \mathrm{mg} /$ week for 26 weeks was evaluated in high-risk patients intolerant to statins. ${ }^{39} \mathrm{LDL}$ cholesterol decreased by $47.3 \%$, with a similar decrease in apolipoprotein B (46.2\%) and a decrease of lipoprotein(a) by $27.1 \%$. Eighteen percent of the mipomersen-treated patients and $17 \%$ of the placebo-treated patients discontinued due to adverse events. As in statin-tolerant patients, injection site reactions and flu-like symptoms were the most common side effects. Liver function tests were above three times the upper limit of normal in 33\% of mipomersentreated patients.

\section{Efficacy of mipomersen}

Mipomersen results in a dose-dependent reduction of LDL cholesterol, lipoprotein(a), apolipoprotein B100, and triglycerides. At the dose chosen for further development (200 mg/week), LDL cholesterol reduction varies between $30 \%$ and $47 \%$ compared with placebo. Apolipoprotein B and lipoprotein(a) reduction is slightly less at 20\%-35\%. Triglyceride reduction is more variable and usually between $10 \%$ and $20 \%$. Within each study, there is considerable variability in the reduction of LDL cholesterol, for example, in homozygous FH, LDL reduction varied between $2 \%$ and $82 \%$. If reported, the treatment effect was independent of baseline LDL cholesterol values, age, race, or gender. The observed reduction in LDL cholesterol, lipoprotein(a), and apolipoprotein B seems to be independent of the underlying cause and independent of concomitant drug therapy.

\section{Safety and tolerability}

Mipomersen has been generally well tolerated and had an acceptable safety profile in the Phase II and Phase III studies. The most common adverse events were injection site reactions, flu-like symptoms, and increases in liver function tests, especially alanine aminotransferase.

Injection site reactions occur in most patients (75\%-100\%). These are dose-dependent and characterized by transient, mild to moderate erythema, occurring within 24 hours of drug injection. On subsequent injection, a flare-up of the previous injection site may occur. Injection site reactions are considered an antisense class-related phenomenon also observed with other antisense drugs. However, the exact 


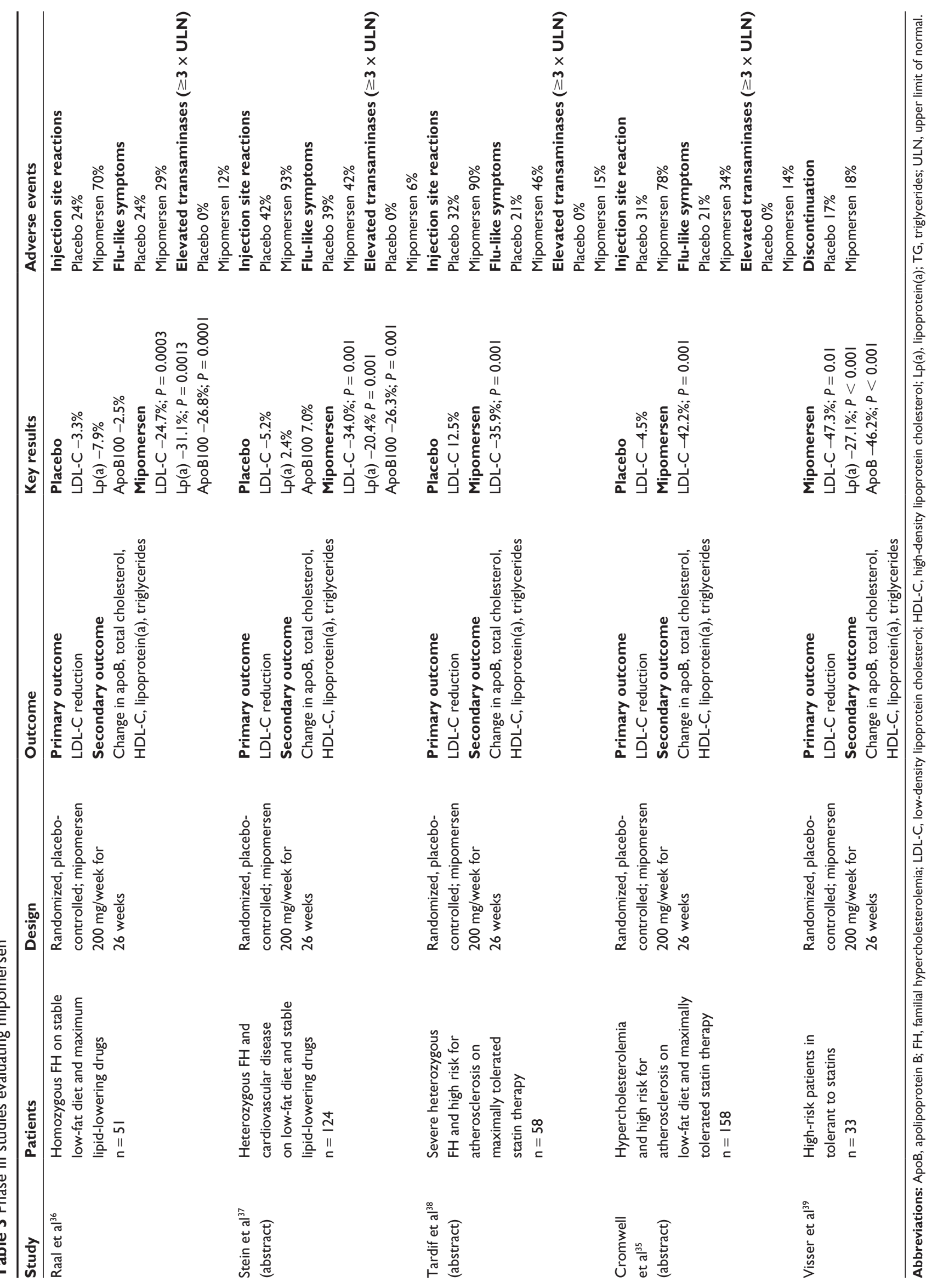


underlying mechanism is unknown. Injection site reactions are not considered to be serious safety concerns but may affect patient compliance.

Flu-like symptoms were reported somewhat more often in the mipomersen groups than in the placebo groups. Elevated transaminases (alanine aminotransferase level more than three times the upper limit of normal) was observed in $6 \%-15 \%$ of mipomersen-treated patients $(0 \%$ in the placebo groups). These elevations were not accompanied by other clinical or laboratory findings suggestive of hepatic dysfunction nor were there any cases of Hy's law. Elevated alanine aminotransferase most likely reflects accumulation of hepatic fat secondary to inhibition of apolipoprotein B synthesis and therefore very-low-density lipoprotein secretion. Hepatic steatosis has been observed in some mipomersen-treated patients. ${ }^{40}$ Hepatic steatosis resolves and alanine aminotransferase elevations return to normal after discontinuation of treatment.

\section{Therapeutic potential of mipomersen}

The currently available studies indicate that mipomersen may decrease LDL cholesterol in homozygous and heterozygous FH patients by approximately $30 \%$, independent of concomitant drug therapy. The effect of mipomersen in patients treated with apheresis is currently unknown, but there is little doubt about similar efficacy in such patients.

Thus, mipomersen could theoretically be used in all patients not at goal with respect to LDL cholesterol and at sufficient absolute risk to justify treatment with a drug for which no long-term data are currently available. Mipomersen may be of particular benefit to patients with homozygous $\mathrm{FH}$ or severe heterozygous FH, given that treatment options are very limited in such patients. It should also be noted that mipomersen, in contrast with other established approaches, such as statins, bile acid-binding resins, and ezetimibe, and in contrast with other newly developed approaches, such as PCSK9 inhibitors, does not rely on the LDL receptor to achieve LDL cholesterol reduction.

The following patient groups may be primary candidates for treatment with mipomersen:

- Patients with homozygous FH, many of whom do not respond to statin therapy and even if they do, usually do not reach treatment goals. In this patient group, a Phase III study has shown efficacy with respect to LDL, apolipoprotein B, and lipoprotein(a) reduction.

- Patients with severe heterozygous FH, who usually respond to statin therapy, with an LDL cholesterol reduction of up to $50 \%$, but may not reach treatment goals either because baseline values are too high or because they do not tolerate high-dose statins. Mipomersen as an addon therapy to a statin (and potentially ezetimibe and bile-acid binding resins) may further reduce LDL cholesterol and result in a considerably larger fraction of patients reaching treatment goals. Such patients have been studied in a Phase III trial, but the results have only been published in abstract form so far.

- Patients with severe hypercholesterolemia and coronary heart disease not reaching treatment goals. Similar to patients with heterozygous $\mathrm{FH}$, these patients usually respond to conventional drug therapy, but efficacy may be inadequate. Addon therapy with mipomersen may allow more patients to reach treatment goals.

- Patients at high risk for atherosclerosis intolerant to statin therapy, in whom mipomersen may be a treatment alternative allowing attainment of treatment goals.

Theoretically, all patients qualifying for regular apheresis should be candidates for mipomersen treatment. In fact, according to German guidelines, a hypothetical evaluation shows that approximately $50 \%$ of aphereses could be avoided if mipomersen was available on the German market and tolerated as well as apheresis. ${ }^{41}$ Obviously, mipomersen may also be an interesting addon therapy to apheresis, which may lead to lower pre-apheresis and post-apheresis values, and thus to better average values. In some patients, this may have the benefit of apheresis being required less frequently. However, before mipomersen can be used as concomitant therapy in apheresis-treated patients, a proper study must be performed in this patient group.

Although mipomersen has a significant ability to lower LDL, apolipoprotein B, and lipoprotein(a), and may thus allow more people to reach therapeutic goals, it also has significant side effects (injection site reactions, elevation of transaminases) which may affect compliance and must be taken into account in cost-benefit analysis. Until more long-term data are available, especially with respect to elevated liver enzymes, this form of therapy will be used primarily in patients with severe forms of hypercholesterolemia and at very high cardiovascular risk.

In summary, inhibition of apolipoprotein B production using mipomersen may be an interesting approach for patients with heterozygous and homozygous FH not at target with current forms of drug therapy. Mipomersen reduces LDL cholesterol by up to $45 \%$, but leads to injection site reactions in almost all patients, and flu-like symptoms and elevated transaminases in some. 


\section{Disclosure}

KGP has received honoraria for presentations, advisory board activities, and Data Monitoring Committee activities by Abbott, Astra-Zeneca, Boehringer-Ingelheim, Bristol-Myers Squibb, Fresenius, Genzyme, Kaneka, Merck Sharp and Dohme, Roche. KGP has received research support from Genzyme and Merck Sharp and Dohme.

\section{References}

1. Soutar AK, Naoumova RP. Mechanisms of disease: genetic causes of familial hypercholesterolemia. Nat Clin Pract Cardiovasc Med. 2007;4(4):214-225.

2. Brown MS, Goldstein JL. Familial hypercholesterolemia: defective binding of lipoproteins to cultured fibroblasts associated with impaired regulation of 3-hydroxy-3-methylglutaryl coenzyme A reductase activity. Proc Natl Acad Sci U S A. 1974;71(3):788-792.

3. Goldstein JL, Brown MS. Familial hypercholesterolemia: identification of a defect in the regulation of 3-hydroxy-3-methylglutaryl coenzyme A reductase activity associated with overproduction of cholesterol. Proc Natl Acad Sci U S A. 1973;70(10):2804-2808.

4. Gaffney D, Reid JM, Cameron IM, et al. Independent mutations at codon 3500 of the apolipoprotein B gene are associated with hyperlipidemia Arterioscler Thromb Vasc Biol. 1995;15(8):1025-1029.

5. Soria LF, Ludwig EH, Clarke HR, Vega GL, Grundy SM, McCarthy BJ. Association between a specific apolipoprotein B mutation and familial defective apolipoprotein B100. Proc Natl Acad Sci U S A. 1989;86(2): 587-591.

6. Abifadel M, Varret M, Rabes JP, et al. Mutations in PCSK9 cause autosomal dominant hypercholesterolemia. Nat Genet. 2003;34(2):154-156.

7. Cohen JC, Boerwinkle E, Mosley TH Jr, Hobbs HH. Sequence variations in PCSK9, low LDL, and protection against coronary heart disease. N Engl J Med. 2006;354(12):1264-1272.

8. Robinson JG, Goldberg AC. Treatment of adults with familial hypercholesterolemia and evidence for treatment: recommendations from the National Lipid Association Expert Panel on Familial Hypercholesterolemia. J Clin Lipidol. 2011;5(Supp1 3):S18-S29.

9. Sjouke B, Kusters DM, Kastelein JJ, Hovingh GK. Familial hypercholesterolemia: present and future management. Curr Cardiol Rep. 2011;13(6):527-536.

10. Baigent C, Blackwell L, Emberson J, et al. Efficacy and safety of more intensive lowering of LDL cholesterol: a meta-analysis of data from 170,000 participants in 26 randomised trials. Lancet. 2010 376(9753):1670-1681.

11. Baigent C, Keech A, Kearney PM, et al. Efficacy and safety of cholesterol-lowering treatment: prospective meta-analysis of data from 90,056 participants in 14 randomised trials of statins. Lancet. 2005;366(9493):1267-1278.

12. Versmissen J, Oosterveer DM, Yazdanpanah M, et al. Efficacy of statins in familial hypercholesterolaemia: a long term cohort study. BMJ. 2008;337:a2423.

13. Raal FJ, Pilcher GJ, Panz VR, et al. Reduction in mortality in subjects with homozygous familial hypercholesterolemia associated with advances in lipid-lowering therapy. Circulation. 2011;124(20):2202-2207.

14. Elis A, Zhou R, Stein EA. Effect of lipid-lowering treatment on natural history of heterozygous familial hypercholesterolemia in past three decades. Am J Cardiol. 2011;108(2):223-226.

15. Huijgen R, Abbink EJ, Bruckert E, et al. Colesevelam added to combination therapy with a statin and ezetimibe in patients with familial hypercholesterolemia: a 12-week, multicenter, randomized, doubleblind, controlled trial. Clin Ther. 2010;32(4):615-625.

16. Thompson GR. Lipoprotein apheresis. Curr Opin Lipidol. 2010;21(6): 487-491.
17. Thompson GR, Barbir M, Davies D, et al. Efficacy criteria and cholesterol targets for LDL apheresis. Atherosclerosis. 2010;208(2):317-321.

18. American Society For Apheresis. Therapeutic apheresis: a guide to billing and securing appropriate reimbursement, 2006. Available from: http://www.apheresis.org/ ASSETS/DOCUMENT/PDF/Committee/ Therapeutic\%20Apheresis_A\%20Guide $\% 20$ to\%20Billing\%20and $\% 20$ Securing\%20Appropriate\%20Reimbursement\%20(2006).pdf. Accessed April 24, 2012.

19. Kucukkartallar T, Yankol Y, Kanmaz T, Topaloglu S, Acarli K, Kalayoglu M. Liver transplantation as a treatment option for three siblings with homozygous familial hypercholesterolemia. Pediatr Transplant. 2011;15(3):281-284.

20. Brown WV, Bays H, Davidson M, Goldberg A. Drugs in development for management of lipoprotein disorders. $J$ Clin Lipidol. 2011;5(2):66-75.

21. Chan JC, Piper DE, Cao Q, et al. A proprotein convertase subtilisin/kexin type 9 neutralizing antibody reduces serum cholesterol in mice and nonhuman primates. Proc Natl Acad Sci U S A. 2009;106(24): 9820-9825.

22. Frank-Kamenetsky M, Grefhorst A, Anderson NN, et al. Therapeutic RNAi targeting PCSK9 acutely lowers plasma cholesterol in rodents and LDL cholesterol in nonhuman primates. Proc Natl Acad Sci USA. 2008;105(33):11915-11920.

23. Graham MJ, Lemonidis KM, Whipple CP, et al. Antisense inhibition of proprotein convertase subtilisin/kexin type 9 reduces serum LDL in hyperlipidemic mice. J Lipid Res. 2007;48(4):763-767.

24. Konrad RJ, Troutt JS, Cao G. Effects of currently prescribed LDL-Clowering drugs on PCSK9 and implications for the next generation of LDL-C-lowering agents. Lipids Health Dis. 2011;10:38.

25. Ladenson PW, Kristensen JD, Ridgway EC, et al. Use of the thyroid hormone analogue eprotirome in statin-treated dyslipidemia. $N \mathrm{Engl}$ J Med. 2010;362(10):906-916.

26. Barkowski RS, Frishman WH. HDL metabolism and CETP inhibition. Cardiol Rev. 2008;16(3):154-162.

27. Raval SK, Raval PS, Jain MR. Emerging therapies for dyslipidemia: known knowns and known unknowns of MTP inhibitors. Recent Pat Endocr Metab Immune Drug Discov. 2012;6(1):24-29.

28. Sniderman AD, Williams K, Contois JH, et al. A meta-analysis of low-density lipoprotein cholesterol, non-high-density lipoprotein cholesterol, and apolipoprotein B as markers of cardiovascular risk. Circ Cardiovasc Qual Outcomes. 2011;4(3):337-345.

29. Bennett CF, Swayze EE. RNA targeting therapeutics: molecular mechanisms of antisense oligonucleotides as a therapeutic platform. Annu Rev Pharmacol Toxicol. 2010;50:259-293.

30. Kastelein JJ, Wedel MK, Baker BF, et al. Potent reduction of apolipoprotein B and low-density lipoprotein cholesterol by shortterm administration of an antisense inhibitor of apolipoprotein B. Circulation. 2006;114(16):1729-1735.

31. Yu RZ, Geary RS, Flaim JD, et al. Lack of pharmacokinetic interaction of mipomersen sodium (ISIS 301012), a 2'-O-methoxyethyl modified antisense oligonucleotide targeting apolipoprotein B100 messenger RNA, with simvastatin and ezetimibe. Clin Pharmacokinet. 2009;48(1):39-50.

32. Akdim F, Visser ME, Tribble DL, et al. Effect of mipomersen, an apolipoprotein B synthesis inhibitor, on low-density lipoprotein cholesterol in patients with familial hypercholesterolemia. Am J Cardiol. 2010;105(10):1413-1419.

33. Akdim F, Stroes ES, Sijbrands EJ, et al. Efficacy and safety of mipomersen, an antisense inhibitor of apolipoprotein B, in hypercholesterolemic subjects receiving stable statin therapy. J Am Coll Cardiol. 2010;55(15):1611-1618.

34. Akdim F, Tribble DL, Flaim JD, et al. Efficacy of apolipoprotein B synthesis inhibition in subjects with mild-to-moderate hyperlipidaemia. Eur Heart J. 2011;32(21):2650-2659.

35. Cromwell WC, Thomas GS, Boltje I, Chin W, Davidson M. Safety and efficacy of mipomersen administered as add-on therapy in patients with hypercholesterolemia and high cardiovascular risk. J Am Coll Cardiol. 2011:Abstract. 
36. Raal FJ, Santos RD, Blom DJ, et al. Mipomersen, an apolipoprotein B synthesis inhibitor, for lowering of LDL cholesterol concentrations in patients with homozygous familial hypercholesterolaemia: a randomised, double-blind, placebo-controlled trial. Lancet. 2010; 375(9719):998-1006.

37. Stein E, Dufour R, Gagne C, et al. A randomized, double-blind, placebo-controlled study to assess efficacy and safety of mipomersen as add-on-therapy in herterozygous familial hyoercholesterolemia patients wirh coronary artery disease. Eur Heart J. 2010; 31 Suppl 1:Abstract.

38. Tardif JC, Ceska R, Burgess LJ, et al. Apolipoprotein B-synthesis inhibition by mipomersen reduces LDL-C when added to maximally tolerated lipid-lowering medication in patients with severe heterozygous hypercholesterolemia. J Am Coll Cardiol. 2011:Abstract.
39. Visser ME, Wagener G, Baker BF, et al. Mipomersen, an apolipoprotein B synthesis inhibitor, lowers low-density lipoprotein cholesterol in high-risk statin-intolerant patients: a randomized, double-blind, placebo-controlled trial. Eur Heart J. April 16, 2012. [Epub ahead of print.]

40. Visser ME, Akdim F, Tribble DL, et al. Effect of apolipoprotein-B synthesis inhibition on liver triglyceride content in patients with familial hypercholesterolemia. J Lipid Res. 2010;51(5):1057-1062.

41. Parhofer KG, Vogt A. 760 mipomersen and apoB synthesis inhibitor evaluation of potential to reduce necessity for lipid-apheresis in patients with heterozygous FH and CAD. Atherosclerosis. 2011;12(1):159.

\section{Publish your work in this journal}

Core Evidence is an international, peer-reviewed open-access journal evaluating the evidence underlying the potential place in therapy of drugs throughout their development lifecycle from preclinical to postlaunch. The focus of each review is to evaluate the case for a new drug or class in outcome terms in specific indications and patient groups.

\section{Dovepress}

The manuscript management system is completely online and includes a very quick and fair peer-review system, which is all easy to use. Visit http://www.dovepress.com/testimonials.php to read real quotes from published authors. 